\title{
Validation of X-ray lithography and development simulation system for moving mask deep $\mathrm{X}$-ray lithography
}

\section{AUTHOR(S):}

Hirai, Y; Hafizovic, S; Matsuzuka, N; Korvink, JG; Tabata, $\mathrm{O}$

\section{CITATION:}

Hirai, Y ... [et al]. Validation of X-ray lithography and development simulation system for moving mask deep X-ray lithography. JOURNAL OF MICROELECTROMECHANICAL SYSTEMS 2006, 15(1): 159-168

\section{ISSUE DATE:}

2006-02

URL:

http://hdl.handle.net/2433/50210

\section{RIGHT:}

(c)2006 IEEE. Personal use of this material is permitted. However, permission to reprint/republish this material for advertising or promotional purposes or for creating new collective works for resale or redistribution to servers or lists, or to reuse any copyrighted component of this work in other works must be obtained from the IEEE. 


\title{
Validation of X-Ray Lithography and Development Simulation System for Moving Mask Deep X-Ray Lithography
}

\author{
Yoshikazu Hirai, Sadik Hafizovic, Naoki Matsuzuka, Jan G. Korvink, and Osamu Tabata, Senior Member, IEEE
}

\begin{abstract}
This paper presents a newly developed 3-Dimensional (3-D) simulation system for Moving Mask Deep X-ray Lithography $\left(\mathrm{M}^{2} \mathrm{DXL}\right)$ technique, and its validation. The simulation system named X-ray Lithography Simulation System for 3-Dimensional Fabrication (X3D) is tailored to simulate a fabrication process of 3-D microstructures by $\mathrm{M}^{2} \mathrm{DXL}$. X3D consists of three modules: mask generation, exposure and resist development (hereafter development). The exposure module calculates a dose distribution in resist using an X-ray mask pattern and its movement trajectory. The dose is then converted to a resist dissolution rate. The development module adopted the "Fast Marching Method" technique to calculate the 3-D dissolution process and resultant 3-D microstructures. This technique takes into account resist dissolution direction that is required by 3-D X-ray lithography simulation. The comparison between simulation results and measurements of "stairs-like" dose deposition pattern by $\mathrm{M}^{2} \mathrm{DXL}$ showed that X3D correctly predicts the 3-D dissolution process of exposed PMMA.

[1474]
\end{abstract}

Index Terms-Microfabrication, simulation, three-dimensional (3-D), X-ray lithography.

\section{INTRODUCTION}

$\mathbf{T}$ HE LIGA process employs Deep X-Ray Lithography (DXRL) to produce metal or plastic microstructures that feature sizes down to $0.1 \mu \mathrm{m}$. In order to realize such a microstructure, a thick resist is first exposed to Synchrotron Radiation (SR) through an X-ray mask and the exposed resist is developed. By combining DXRL with subsequent electroplating and molding, replication of the fabricated microstructures in various materials such as plastics, metals and ceramics become possible. Conventional DXRL is good at

Manuscript received December 1, 2004; revised March 29, 2005. This work was supported in part by Center of Excellence for Research and Education on Complex Functional Mechanical Systems (COE program of the Ministry of Education, Culture, Sports, Science and Technology, Japan). Subject Editor H. Fujita.

Y. Hirai is with the Department of Micro Engineering, Graduate School of Engineering, Kyoto University, Kyoto 606-8501, Japan (e-mail: hirai@sens.mbox.media.kyoto-u.ac.jp).

S. Hafizovic is with the Physical Electronics Laboratory, Institute for Quantum Electronics, Swiss Federal Institute of Technology (ETH) Zürich, 8093 Zürich, Switzerland (e-mail: hafizovi@phys.ethz.ch).

N. Matsuzuka was with the Graduate School of Science and Engineering, Ritsumeikan University, Noji-Higashi, Kusatsu, Shiga 525-8577, Japan. He is now with the Department of Micro Engineering, Graduate School of Engineering, Kyoto University, Kyoto 606-8501, Japan (e-mail: matsuzuka@mem.iee.or.jp).

J. G. Korvink is with the IMTEK-Institute of Microsystem Technology, University of Freiburg, D-79110 Freiburg, Germany (e-mail: korvink@imtek.de).

O. Tabata is with the Department of Micro Engineering, Graduate School of Engineering, Kyoto University, Kyoto 606-8501, Japan (e-mail: tabata@me.kyoto-u.ac.jp).

Digital Object Identifier 10.1109/JMEMS.2005.859191 fabricating microstructures with vertical sidewalls and high-aspect ratio microstructures. However, this technique has very limited controllability of the cross-sectional shape of three-dimensional (3-D) microstructures. In order to apply DXRL to various fields such as microsensors, microactuators and MEMS devices, more flexible and precise controllability of the 3-D microstructure is demanded. To address these requirements, several techniques have been proposed such as Moving Mask Deep X-ray Lithography ( $\mathrm{M}^{2} \mathrm{DXL}$ ) technique [1], its extension [2] and inclined exposure technique [3]. $\mathrm{M}^{2} \mathrm{DXL}$ technique shown in Fig. 1 is one of the highly promising 3-D X-ray lithography techniques to realize 3-D microstructures with free shaped and inclined walls [1], [4], [5]. In $\mathrm{M}^{2} \mathrm{DXL}$, the 3-D microstructure is defined by an X-ray mask trajectory and the resultant dose distribution in resist.

On the other hand, very little attention has been paid to "3-D" X-ray lithography simulation in these techniques. Meyer et al. [6] have focused on the determination of an optimal set of parameters for an X-ray lithography beamline and resist development (hereafter development) condition. Griffiths et al. [7] have studied the dose distribution near an X-ray mask absorber edge due to photoelectron dose and the effects of this distribution on the 2-D time series of development profile with analytical and numerical methods. Hagouel [8] models theoretically the development process of X-ray lithography and he solved the Hamilton-Jacobi equation by applying ray tracing techniques. However, there is little adaptability for 3-D X-ray lithography techniques because these studies do not cover 3-D geometrical aspects in the simulation. Another study on 3-D development simulation developed by Bollepalli et al. [9] has targeted a thin resist geometry handling since this investigation was motivated by microelectronics manufacturing. Consequently, there has been no study of 3-D simulation system that covers the complete process of 3-D X-ray lithography technique including both X-ray exposure and development process of 3-D microstructures as time series.

We have reported in previous work [10] that the dose distribution in resist and the resist dissolution direction are not sufficient to correctly calculate a 3-D dissolution process and resultant 3-D microstructures. Therefore, our target simulation system necessarily should take into account an X-ray exposure with $\mathrm{M}^{2} \mathrm{DXL}$ technique and a development process. Based on this consideration, an X-ray lithography simulation system named X-ray Lithography Simulation System for 3-Dimensional Fabrication $(\mathrm{X} 3 \mathrm{D})$ was newly developed [11]. X3D calculates the 


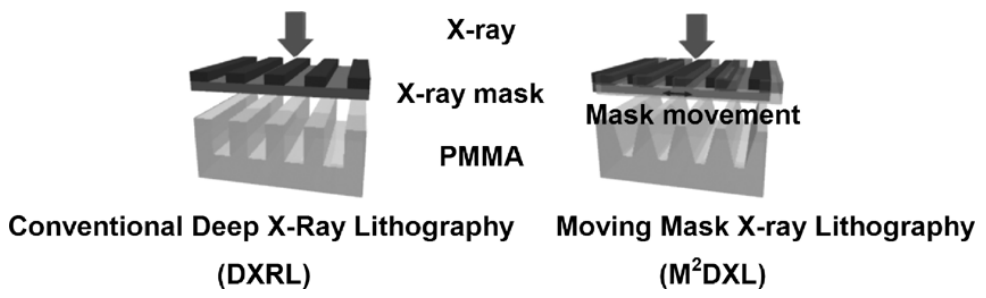

Fig. 1. Concept of $\mathrm{M}^{2} \mathrm{DXL}$.

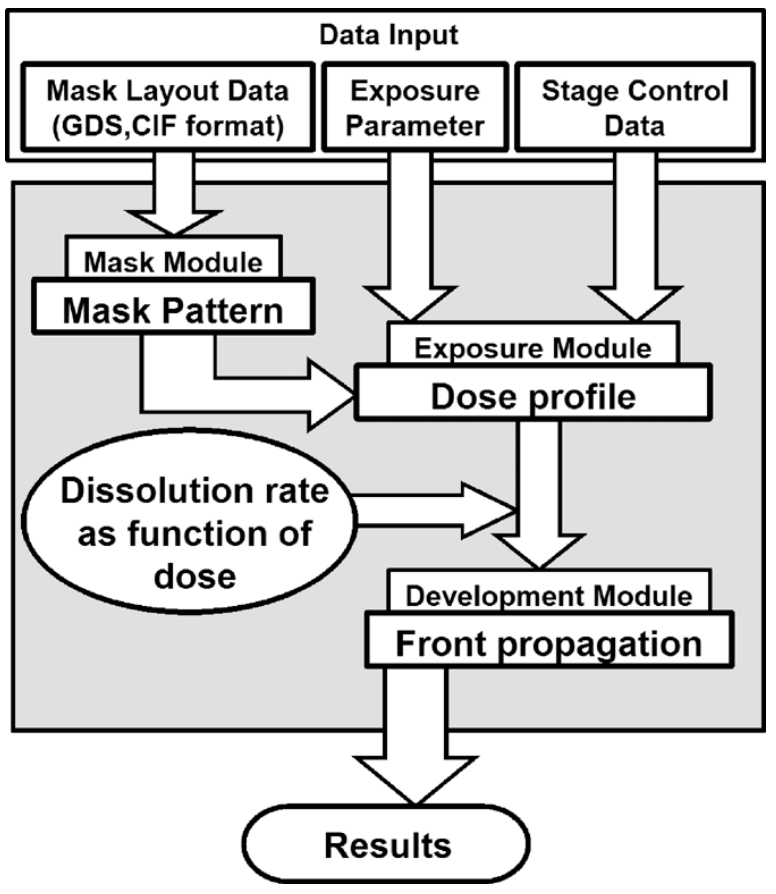

Fig. 2. Data flow in X3D.

propagation of the dissolution front and resist dissolution direction as required for 3-D X-ray lithography simulation. Therefore, X3D enabled to simulate the 3-D dissolution process and resultant 3-D microstructures. In this respect, our simulation system differs from previously reported X-ray lithography simulation. From these viewpoints, X3D is tailored to simulate the fabrication process of 3-D microstructures using $\mathrm{M}^{2} \mathrm{DXL}$ and its capabilities are fundamental to put $\mathrm{M}^{2} \mathrm{DXL}$ in practical use. Furthermore, the newly developed X3D enables us to fabricate rapid prototyping of 3-D microstructures and greatly save time and cost for product development. This paper presents the in-detail structure of X3D, components and algorithm for development time series simulation to predict 3-D microstructures, and the verification of simulation results.

\section{DESCRIPTION OF X3D}

\section{A. Architecture of $X 3 D$}

X3D for $\mathrm{M}^{2} \mathrm{DXL}$ consists of three modules shown in Fig. 2: mask generation, exposure and development. The parameters of the SR source and the beamline, the X-ray mask layout data, the multiple stage system movement data, and experimental resist dissolution rate data are major inputs in this simulation system. The mask generation module can interface to multiple CAD database formats such as GDS-II and CIF. The exposure module models the X-ray lithography beamline components (filters and an X-ray mask), as well as X-ray mask movement on the multiple stage system. Here, transmission and absorption values of filter and X-ray mask materials required for dose calculation are retrieved from an optical properties database in X3D. To find out the resist dissolution rate at some location, X3D first calculates dose and then converts this dose to a rate by means of experimentally measured dose dissolution rate relationship. The development module adopted the Fast Marching Method [12], as described later in 3.3 to calculate the resultant 3-D microstructure based on the experimental resist dissolution rate.

All of X3D's results, such as X-ray spectra in resist and dose distribution for any spatial region in the simulation domain are readable in Mathematica ${ }^{\mathrm{TM}}$ for further analysis. Additionally, geometrical data of a resultant 3-D microstructure at an arbitrary time is extracted for visualization in graphical tool, e.g., POV-Ray.

\section{B. Exposure Simulation}

The exposure simulation that calculates dose distribution in resist is composed of X-ray lithography components: the SR source, beamline filters, and the multiple stage system. The dose is defined as the amount of X-ray energy absorbed per unit volume and relates to the resist solubility into a developer [13], [14]. So the dose calculation should take into account the SR spectrum, X-ray transmission through filters and the X-ray mask membrane, photon absorption in resist, and also account for the $\mathrm{X}$-ray mask movement on the multiple stage system.

Here, we introduce the plane corresponding to the initial resist surface as $x-y$ plane, and the resist depth from the surface $(z=$ 0 ) down to the bottom as $z$. In case of 3-D X-ray lithography techniques including $\mathrm{M}^{2} \mathrm{DXL}$, dose has a variation not only in $z$ direction but also in the $x-y$ plane. Then at a specific point on the resist surface $(x, y)$, the dose "Dose $(x, y, z)$ " as a function of $z$ can be expressed as [15], [16]

$\operatorname{Dose}(x, y, z)=T_{E}(x, y) I \int N(\lambda) B(\lambda) A(\lambda, z) T_{F}(\lambda) T_{M}(\lambda) d \lambda$

where

$\lambda$ :

$T_{E}(x, y): \quad$ effective exposure time determined in ac-

$I$ :

$N(\lambda)$ :

$B(\lambda)$ : cordance with X-ray mask trajectory; stored electron beam current; photon flux spectral distribution through per unit area; conversion factor from photon to energy units; 


$$
\begin{array}{ll}
A(\lambda, z): & \begin{array}{l}
\text { absorption coefficient per unit length of } \\
\text { the resist at depth } z ;
\end{array} \\
T_{F}(\lambda), T_{M}(\lambda): & \begin{array}{l}
\text { transmission coefficients of filter } T_{F} \text { and } \\
\text { X-ray mask membrane } T_{M} .
\end{array}
\end{array}
$$

More accurate descriptions of the SR source, photon flux calculation, and the dose calculation will not be discussed in this paper (see in [6] and [15]).

\section{Development Simulation}

The development simulation, calculates the resist dissolution process based on the results of exposure simulation, and relies on the Fast Marching Method. This simulation technique will be discussed in detail in the following chapter. In order to calculate the 3-D dissolution process only on the demanded simulation domain sizes, an "adaptive refinement" technique for fast marching method was developed. This technique allows us to overcome memory restrictions and improve computational speed. In case of finite difference method (FDM) and finite element method (FEM), refinement is done for the whole mesh of the simulation domain before calculation. With the fast marching method in contrast, the adaptive refinement is done locally in space and time while the resist dissolution process is being calculated. Thereby, X3D made it possible to simulate realistic MEMS devices of sizes in the order of $\mathrm{mm}$ on a standard engineering workstation, e.g., the simulation of a domain size of $10 \mathrm{~mm} \times 5 \mathrm{~mm} \times 0.2 \mathrm{~mm}=10 \mathrm{~mm}^{3}$ with a minimum detail size of $0.5 \mu \mathrm{m}$ in $6 \mathrm{~h}$. The detailed discussion of X3D capability and framework are communicated in a separate publication [11].

\section{Mathematical Model For DeVelopment Simulation}

\section{A. Physical and Mathematical Principles}

At any point of development time in the lithography development process, the interface between developer and undeveloped resist is defined as a boundary. The dose distribution is defined as a function of position by (1) and the resist dissolution rate is defined as a function of dose. From these relations, the dissolution rate is defined as a function of position and the developer-resist interface propagation is dominated by this dissolution rate. If there is a dose distribution only perpendicular to the surface (i.e., this is the case for conventional DXRL), the dissolution front moves perpendicularly only. On the other hand, if there is also a lateral dose gradient (i.e., in case of 3-D X-ray lithography), the dissolution rate vector of the front is not perpendicular to the initial surface anymore; namely the dissolution front tends to move faster to the higher dose direction. The problem posed is how to model the propagation of the dissolution front and predict its profile and position at any moment in the development process.

Apart from the development simulation in X-ray lithography, various etching and development simulation techniques have been reported such as "marker/string methods [17]" and "cell removal methods [18]" adapted for anisotropic etching and photo resist development. However there are some drawbacks for these methods when applied to the 3-D X-ray lithography techniques. For example, the marker/string method suffers from instability and topological limitation because it cannot take into account the nature of the dissolution front that once a point is crossed by the dissolution front, it remains crossed (i.e., the dissolution front always moves "one-way"). In case of the cell removal methods, a larger number of cells are required to obtain the same simulation accuracy since the approximation to the dissolution front propagation through cell volume is relatively rough. Additionally, calculation of geometric properties of the dissolution front such as curvature are inaccurate. In order to address the complicated modeling situation of $\mathrm{M}^{2} \mathrm{DXL}$ and perform the simulation accurately, we adopted the fast marching method which has following advantage in development simulation.

\section{B. Level Set Method and Fast Marching Method}

There are two numerical techniques to track a moving of 3-D structures interface, "Level Set Method" and "Fast Marching Method," introduced by Sethian [12]. These techniques cover a wide range of applications including problems of optics, seismology, path planning, robotic navigation, and fluid mechanics. Although they are fundamentally "different approaches" to address the problem of moving interface tracking, a common theory and numerical methodology are utilized in terms of moving interface tracking. The Level Set Method is developed for problems where an interface moves forward in some places and backward in others. The Fast Marching Method is designed for problems where an interface always moves in one direction, either forward or backward. Due to this constraint, the Fast Marching Method is significantly faster and computationally cheaper than the Level Set Method. Development process has the property that the resist dissolution front always moves "one-way." In other words, resist always dissolves and cannot be deposited. For this reason, the Fast Marching Method was applied to our problem.

\section{Fast Marching Propagation}

Before starting with the explanation of the Fast Marching propagation, we introduce some symbols associated to the dissolution front tracking.

$T: \quad$ arrival time of dissolution front at either grid points; ת.SIM: $\quad$ the simulation domain, normally $\subset \Re^{2}$ or $\subset \Re^{3}$;

$\Gamma$ and dissolution front and initial dissolution front (i.e., $\Gamma_{0}: \quad$ initial resist surface), respectively.

$v$ : $\quad$ scalar dissolution rate, only defined on the dissolution front;

$n$ : $\quad$ spatial dimension, normally either 2 or 3.

1) Equation of the Resist Dissolution Front: In the fast marching method, the gradient operator takes a central role to calculate the dissolution front propagation. Given the scalar dissolution rate $v$ that depends on the local position and a suitable gradient definition, the Fast Marching algorithm can construct a solution $T$ which satisfies (2)

$$
\begin{aligned}
|\nabla T|^{-1} & =v \\
T & =\text { const on } \Gamma_{0} .
\end{aligned}
$$

Here, $\Gamma_{0}$ is the initial location of dissolution front. Note that the dissolution rate $v$ will only be evaluated on $\Gamma$ and therefore $v$ needs to only be defined on $\Gamma$. This is the main reason why the 


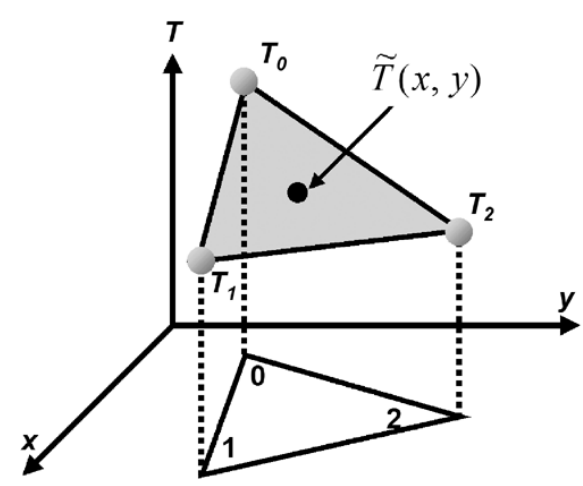

(a)

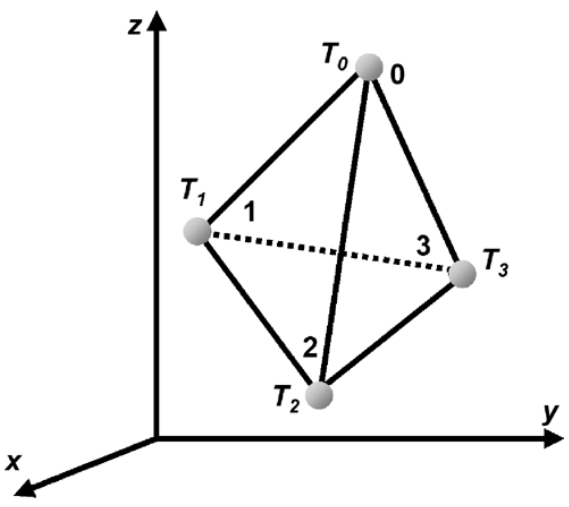

(b)

Fig. 3. (a) Example of triangular element and the linear interpolation of the field $T$ on the element: the number $0,1,2$ are node and $T_{0}, T_{1}, T_{2}$ are the values at the nodes. (b) shows the element for the three-dimensional case.

fast marching method is computationally cheaper for the resist dissolution problem than the level set method.

2) Front Propagation Algorithm: The fast marching method classifies grid points in the simulation domain into three groups: "known" points, "active" points and "unknown" points. The "known" points are points at which the values $T$ are known. The "active" points are points that are not yet known, but are the neighbors of the "known" points. All remaining points are categorized as "unknown" points. Namely, these are the points in the resist except for the interface. The following algorithm changes these "unknown" points first to "active" and eventually to "known" points.

Input: Dissolution rate $v$ on the interface $\Gamma$ and the points defining the initial resist surface $\Gamma_{0}$.

Output: Arrival time $T$ at every grid point in $\Omega \cdot$ SIM .

Procedure:

Step 1. Define the grid points at the resist surface and the adjacent area in the resist.

Step 2. Set $T=0$ for the grid point corresponding to the resist surface, and name the grid points of $\Gamma_{0}$ as "known".

Step 3. All the neighbors of $\Gamma_{0}$ are given initial estimate values for $T$ by solving

(2), and named "active".

Step 4. Set $T=$ infinity (i.e., sufficiently large value) for all remaining grid points and name as "unknown".

Step 5. For all the neighbors of "active" that are not "known," do 5.1, 5.2, and 5.3 .

5.1 Among all the "active" points, extract the point $A$ with the minimum arrival time $T$, and name this point as "known".

5.2 All the neighbors of $A$ of "unknown" are renamed from "unknown" to "active."

5.3 Calculate the value of $T$ at all "active" neighbors of $A$ by solving (2).
Step 6. If all the grid points become "known", or the smallest value $T$ of known point is greater than some time hold, then stop. Otherwise go to step 5.

\section{Triangulated Fast Marching Update Scheme in X3D}

1) Triangulated Fast Marching Method: The nature of the development simulation asks for adaptive refinement of discretization elements. Large areas of uniform dose deposition can be modeled with very few elements, while sharp contrasts along edges and corners demand a very high number of elements to model e.g., edge round-off. Adaptive refinement on a simplex-based (3-D: tetrahedral-based) algorithm is inherently simpler than on higher order elements. For this reason we abandon the classical Fast Marching Method [12] and use a triangulated update scheme.

Even though a triangulated update scheme by Sethian for 2-D exists [12], there is none for 3-D. The 2-D triangulated scheme is based upon trigonometric considerations and relies on trigonometric functions to solve (2). An extension to 3-D is doable, but computationally expensive and has not been presented so far. For these reasons, we applied methods of FEM to solve (2) and found an explicit solution which consists of simple arithmetic expressions only.

2) FEM-Like Gradient Approximation: In order to solve (2), we should consider the gradient operator on $T$ within a triangulated element constructed by adaptive refinement. Then we introduce a FEM-like gradient approximation using a linear Lagrange interpolation. In FEM, the simulation domain is divided into many small regions called finite elements, which are triangles or rectangles, and the value of inside point of a finite element is interpolated from the values on the nodes and on the edge of the finite element.

For example, in 2-D the three-node triangular element shown in Fig. 3(a) is specified by the location of its three corner nodes on the $x-y$ plane. The nodes are labeled 0,1 and 2 in counterclockwise direction. The location of the corners are defined by their Cartesian coordinates: $\left(x_{j}, y_{j}\right)$ for $j=0,1$, and 2. Let $T_{0}$, $T_{1}$ and $T_{2}$ be the values at nodes $0,1,2$, respectively. Considering the linear Lagrange interpolation over the triangular do- 
main, the interpolation function $\widetilde{T}$ that represents the values at point $(x, y)$ is represented as

$$
\widetilde{T}(x, y)=\sum_{j=0}^{2} w_{j} T_{j}
$$

where the weights $w_{j}$ for $j=0,1,2$ is called triangular coordinates in the FEM

$$
w_{j}=\frac{\left|\begin{array}{ccc}
1 & x & y \\
1 & x_{j+1} & y_{j+1} \\
1 & x_{j+2} & y_{j+2}
\end{array}\right|}{\left|\begin{array}{lll}
1 & x_{0} & y_{0} \\
1 & x_{1} & y_{1} \\
1 & x_{2} & y_{2}
\end{array}\right|} \quad 0 \leq w_{j} \leq 1
$$

We use the cyclic notation between $j, j+1$, and $j+2$ :

$$
\begin{aligned}
& \text { when } j=0, \text { then } j+1=1 \text { and } j+2=2 ; \\
& \text { when } j=1 \text {, then } j+1=2 \text { and } j+2=0 ; \\
& \text { when } j=2 \text {, then } j+1=0 \text { and } j+2=1 .
\end{aligned}
$$

The construction of the linear Lagrange interpolation for variables in 3-D follows the same way as in the 2-D. In the 3-D case, the element is a tetrahedron as indicated in Fig. 3(b). As there are four vertices, the matrices of (4) become $4 \times 4$ matrices. Let $\left(x_{j}, y_{j}, z_{j}\right)$ for $j=0,1,2,3$ are the $(x, y, z)$ coordinates of the element nodes $0,1,2,3$. And let $T_{0}, T_{1}, T_{2}$ and $T_{3}$ are the values at nodes $0,1,2$ and 3 , respectively

$$
\begin{aligned}
\widetilde{T}(x, y, z) & =\sum_{j=0}^{3} w_{j} T_{j} \\
w_{j} & =\frac{\left|\begin{array}{cccc}
1 & x & y & z \\
1 & x_{j+1} & y_{j+1} & z_{j+1} \\
1 & x_{j+2} & y_{j+2} & z_{j+2} \\
1 & x_{j+3} & y_{j+3} & z_{j+3}
\end{array}\right|}{\left|\begin{array}{cccc}
1 & x_{0} & y_{0} & z_{0} \\
1 & x_{1} & y_{1} & z_{1} \\
1 & x_{2} & y_{2} & z_{2} \\
1 & x_{3} & y_{3} & z_{3}
\end{array}\right|} 0 \leq w_{j} \leq 1 .
\end{aligned}
$$

The cyclic notation between $j, j+1, j+2$, and $j+3$ is

when $j=0$, then $j+1=1, j+2=2$ and $j+3=3$; when $j=1$, then $j+1=2, j+2=3$ and $j+3=0$; when $j=2$, then $j+1=3, j+2=0$ and $j+3=1$; when $j=3$, then $j+1=0, j+2=1$ and $j+3=2$.

For the linear Lagrange interpolation in case of $n$ dimensions, the generalization of (3) and (5) can be written as

$$
\widetilde{T}=\sum_{j=0}^{n} w_{j} T_{j} .
$$

Then, the gradient of (6) is given as

$$
\nabla \widetilde{T}=\sum_{j=0}^{n}\left(\nabla w_{j}\right) T_{j}
$$

3) General Solution for Arrival Time T: Now, we extend (7) to the triangulated fast marching method. Equation (2) is further evaluated to the following:

$$
\sum_{i=1}^{n}\left(\partial_{i} T\right)^{2}=F^{-2}
$$

Inserting (7) into (8) yields the condition

$$
\sum_{i=1}^{n}\left(\sum_{j=0}^{n}\left(\partial_{i} w_{j}\right) T_{j}\right)^{2}=F^{-2} .
$$

Since the interpolation is piecewise linear, its derivative is piecewise constant. Therefore we may substitute the derivative by a constant: $\partial_{i} w_{j} \rightarrow w_{i, j}$. In other words, $w_{i, j}$ denotes the weight of the $j$ th vertex, and then

$$
\sum_{i=1}^{n}\left(\sum_{j=0}^{n} w_{i, j} T_{j}\right)^{2}=F^{-2}
$$

The goal is to update the value of $T_{0}$ using remaining $T_{1} \ldots T_{j}$, since $T_{0}$ is "active" and $T_{1} \ldots T_{j}$ are "known" in the front propagation algorithm. By solving (10), the general, physically meaningful solution for $T_{0}$ is given for the first time

$$
\begin{aligned}
T_{0} & =\frac{-F A+\sqrt{F^{2} A^{2}+B\left(1-F^{2} \sum_{i=1}^{n}\left(\sum_{j=1}^{n} T_{j} w_{i, j}\right)^{2}\right)}}{F B} . \\
A & =\sum_{i=1}^{n}\left(w_{i, 0} \sum_{j=1}^{n} T_{j} w_{i, j}\right) \\
B & =\sum_{i=1}^{n} w_{i, 0}^{2} .
\end{aligned}
$$

Note that, (11) is valid for $n$ dimensions and free of trigonometric and computationally expensive functions. Based on (11), the adaptive refinement fast marching method for X3D computes $T_{0}$ in 3-D space $(n=3)$. Once the arrival time at the simplices' vertices are computed, (5) provides the Lagrange interpolated arrival time for any point in space. Contouring the volume through an arbitrary time provides the respective development front.

\section{SYSTEM VALIDATION AND Discussion}

\section{A. Stairs-Like Dose Deposition Pattern}

To demonstrate a system validation of the developed X3D, a "stairs-like" dose deposition pattern shown in Fig. 4 was chosen. Despite of the dose deposition pattern's geometrical simplicity, both necessity and validity of X3D which takes into account "local properties" discussed in 3.1, i.e., a dose distribution in resist and a resist dissolution direction, to calculate correctly the 3-D dissolution process are clearly shown. The unique feature of the stairs-like pattern can be understood as follows. As shown in Fig. 5, the dissolution front moves faster in areas of high dose than in areas of low dose. Where the high and low dose 


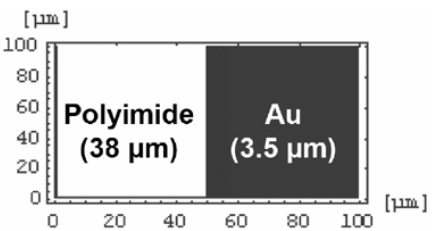

(a)

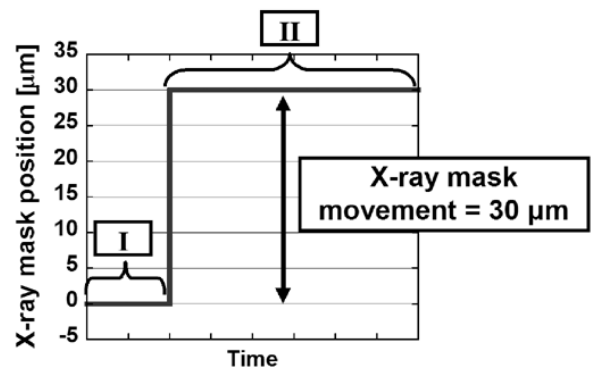

(b)
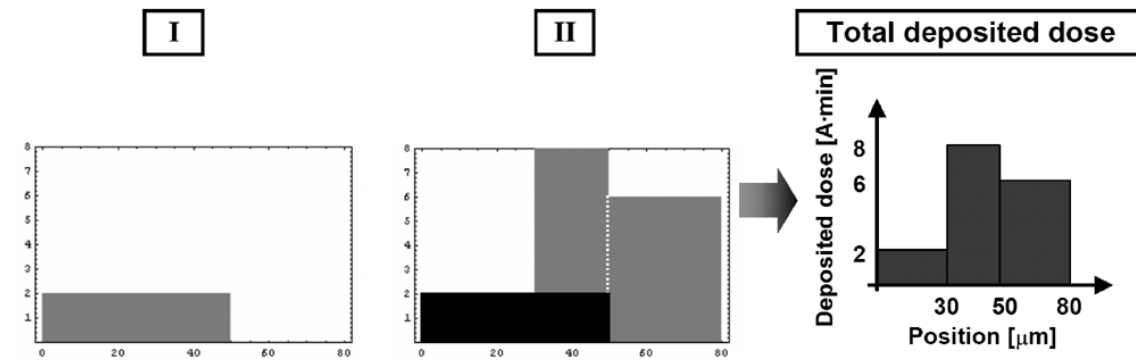

(c)

Fig. 4. (a) The X-ray mask pattern. (b) The X-ray mask movement pattern of the "stairs-like" dose deposition pattern. (c) The deposited dose given by M² DXL In Fig. 4(c), I and II show the dose deposited by the corresponding phases I and II in Fig. 4(b).

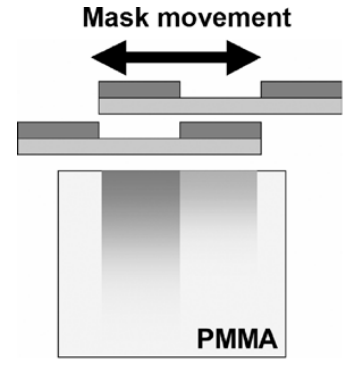

(a)

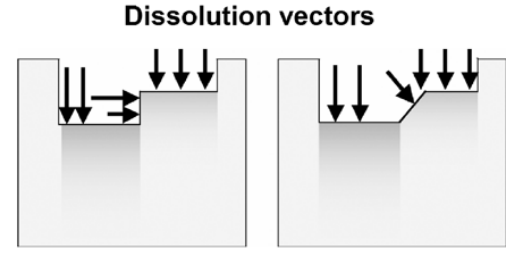

(b)

Fig. 5. (a) shows the dose profile deposited by $\mathrm{M}^{2} \mathrm{DXL}$ and (b) shows the dissolution direction and rounded off edge. In this development process, the dissolution direction is affected by the dose distribution in resist.

regions meet, a step is formed. Since development is isotropic, the exposed sidewall is also subject to dissolution. This leads to rounding of corners and sidewall inclination. If a simulation system does not consider this local dissolution phenomenon, the obtained result becomes unreliable, i.e., the sidewall shape is vertical and the rounded off corner is not obtained.

The stairs-like pattern was deposited into the resist (PMMA: Poly-methylmethacrylate, CLAREX with $1.0 \mathrm{~mm}$ thickness commercialized by Nitto Jushi Kogyo Co., Ltd.) surface using an X-ray mask with a window width of $50 \mu \mathrm{m}$ and a mask movement of $30 \mu \mathrm{m}$ by $\mathrm{M}^{2} \mathrm{DXL}$. For the experiment and simulation, a dose of $1 \mathrm{~A} \cdot$ min corresponding to a net X-ray dose of 9.61 $\mathrm{J} / \mathrm{cm}^{2}$ onto the PMMA was deposited. To develop exposed

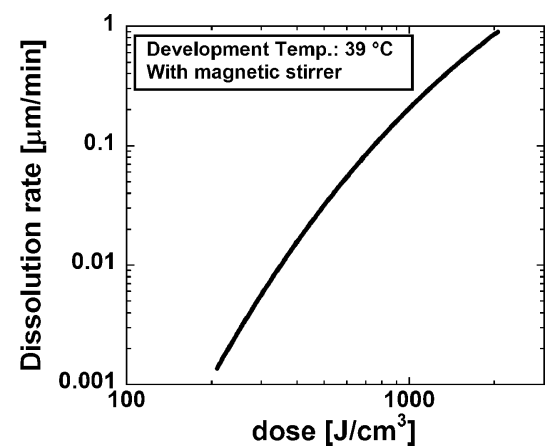

Fig. 6. Experimental dissolution rate as a function of dose.

PMMA samples, GG-developer (15 vol\% DI water, 60 vol\% 2-(2-butoxyethoxy)ethanol, 20 vol\% tetrahydro-1-4-oxazine and $5 \mathrm{vol} \% 2$-aminoethanol) is used at a temperature of $39^{\circ} \mathrm{C}$ with a magnetic stirrer. The following results and discussion are based on the simulation parameters summarized in Table I. The experimental dissolution rate as a function of dose shown in Fig. 6 was used which was extracted in a dedicated experiment series [19].

\section{B. Simulation and Experimental Results}

The exposure simulation results generated by X3D in Fig. 7 illustrate the dose profile in PMMA at three different locations on the sample with irradiation doses of 2.0, 6.0, and 8.0 A - min. Solid lines in Fig. 7 represent the results directly derived from 
TABLE I

SIMULATION PARAMETERS

\begin{tabular}{c|lcr}
\hline \multirow{2}{*}{ SR } & Operating electron energy & $\varepsilon$ & $0.575 \mathrm{GeV}$ \\
(AURORA) & Critical wave length & $\lambda_{\mathrm{c}}$ & $1.5 \mathrm{~nm}$ \\
& Typical source size (vertical) & $\sigma_{\mathrm{y}}$ & $0.14 \mathrm{~mm}$ \\
& Distance between source to mask & $\mathrm{D}$ & $3.88 \mathrm{~m}$ \\
\hline \multirow{2}{*}{ Exposure } & Filter (Be) & & $200 \mu \mathrm{m} \mathrm{x}$ \\
parameter & Scan & $20 \mathrm{~mm}$ \\
& X-ray mask movement & $30 \mu \mathrm{m}$ \\
\hline \multirow{2}{*}{ X-ray mask } & Absorber (Au) & $3.5 \mu \mathrm{m}$ \\
& Membrane (Polyimide) & $38 \mu \mathrm{m}$ \\
\hline Development & Developer & GG-developer \\
parameter & Temperature & $39^{\circ} \mathrm{C}$ \\
\hline
\end{tabular}

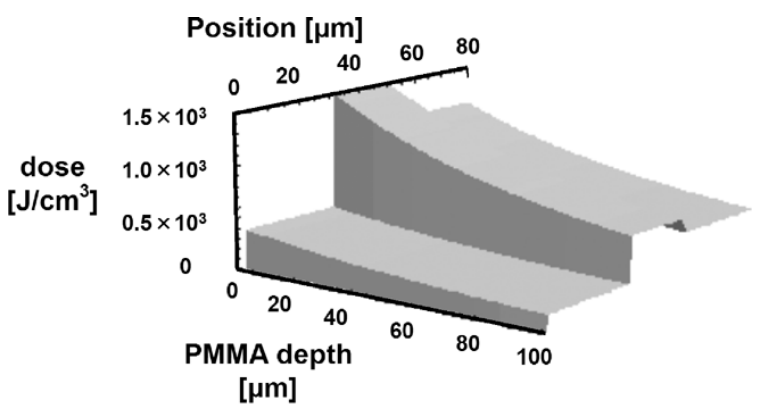

(a)

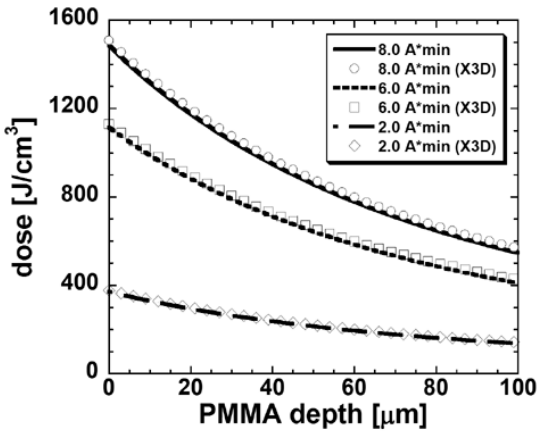

(b)

Fig. 7. 3-D image of dose distributions (a) and simulated dose profiles (b) in PMMA with deposited dose of 2.0,6.0, and $8.0 \mathrm{~A} \cdot \min$. Continuous lines in (b) represent the results derived from (1).

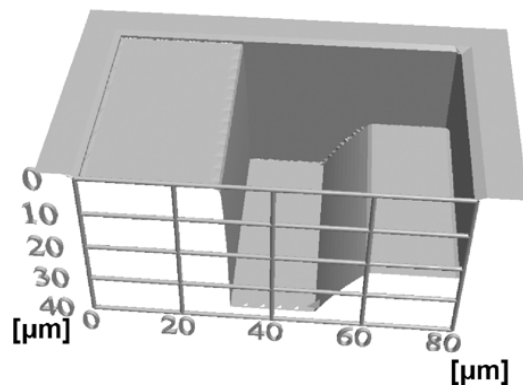

Fig. 8. Simulation result with a development time of $120 \mathrm{~min}$

(1). This indicates that X3D correctly calculates the beamline effects (windows, filters, scanning, and X-ray mask movement) and X-ray absorption in the resist. The calculated dose distributions are accurate. Fig. 8 shows the development simulation result with development time of $120 \mathrm{~min}$.

Fig. 9 shows the experimental result with a development time of $120 \mathrm{~min}$. It should be emphasized that the sidewall shape was not vertical and the corner was rounded off in both Figs. 8 and 9.

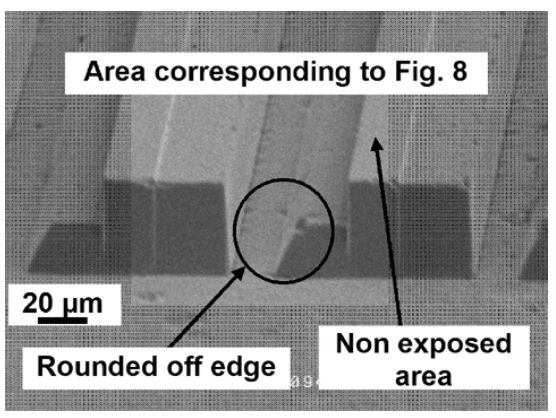

Fig. 9. Experimental result with a development time of $120 \mathrm{~min}$.

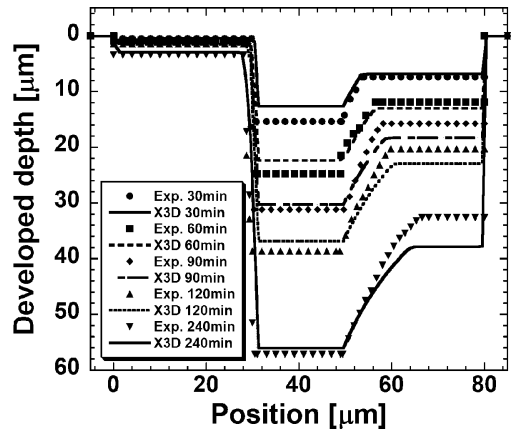

Fig. 10. Comparison of simulation results to measurements with development times of $30,60,90,120$, and $240 \mathrm{~min}$.

This result is attributed to the local dissolution phenomena. The simulation results were evaluated further by comparing with the measured development profiles. Fig. 10 shows the measured results and the development profile extracted from the simulated data of X3D for a sequence of development times. As mentioned above, the same phenomenon of increase in edge roundness was observed both in the experiments and the simulation results. For the first time these 3-D dissolution phenomena from both analytical simulation and experiment are demonstrated.

However, the simulation results slightly deviate from the measurements. There was no significant difference between X3D's calculated dose profile and that obtained by an analytical approach. The accuracy of the triangulated Fast Marching update scheme was also confirmed through a comparison between the calculated results from the X3D development module and those of the analytical approach with the same given flat dose profile. Hence the largest remaining factor is our formulation of the dissolution rate $v$. This suggests that the dissolution rate are either not precise enough or the straight forward translation of dose to dissolution rate is inadequate. This matter is discussed in the next section.

\section{Discussion}

1) Precision of the Experimental Dissolution Rate: In our previous work [20], the accuracy of the measured dissolution rate as a function of dose shown in Fig. 6 was verified through two experiments under the same experimental condition summarized in Table I without X-ray mask movement (i.e., DXRL). Fig. 11(a) shows the dependency of developed depth on development time with an X-ray dose of $7.9 \mathrm{~A} \cdot \min$ (corresponds to a top dose of $1468 \mathrm{~J} / \mathrm{cm}^{3}$ ), and Fig. 11(b) shows the dependency of developed depth on deposited dose (top dose range: 


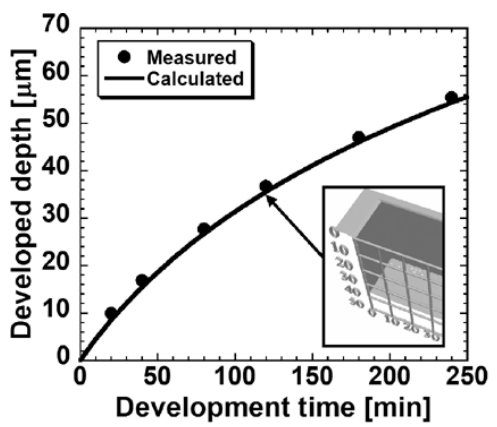

(a)

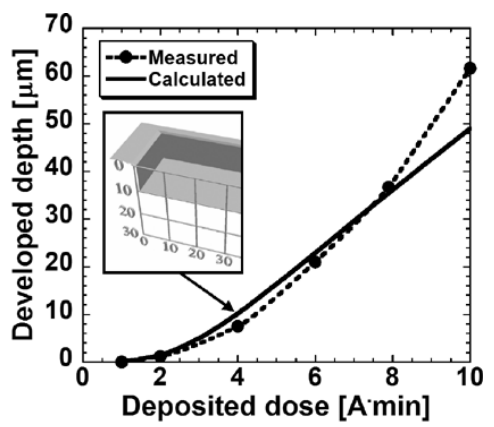

(b)

Fig. 11. (a) Dependency of developed depth on development time with a deposited dose of $7.9 \mathrm{~A} \cdot \mathrm{min}$ and a corresponding 3-D image of X3D with development time of 120 min. (b) Dependency of developed depth on deposited dose with development time of 120 min and the corresponding 3-D image of X3D with a deposited dose of $4.0 \mathrm{~A} \cdot \min$.

186 to $1859 \mathrm{~J} / \mathrm{cm}^{3}$ ) with development time of $120 \mathrm{~min}$. In these experiments, the maximum aspect ratio was 0.04 . According to the verification experiments, the experimental dissolution rate had good accuracy with a dose of around 1400 $\mathrm{J} / \mathrm{cm}^{3}$. On the other hand, the dissolution rate at higher doses than $1400 \mathrm{~J} / \mathrm{cm}^{3}$ (high-dose region) was slightly lower than the actual one, and the dissolution rate at lower doses (low-dose region) was slightly higher than the actual one. Therefore, the developed depth at the high dose region from the position 30 to 50 $\mu \mathrm{m}$ (i.e., $8.0 \mathrm{~A} \cdot \mathrm{min}$ ) in Fig. 10 is calculated lower than experiments. Also, the relative error between experimental and actual dissolution rate $v(\Delta v / v)$ tended to increase with decreasing in dose. As a consequence of the observed dissolution rate behavior and the developed depth calculated by time-integration of the dissolution rate, the difference between simulation and measurement is increasing with time. This is because the front advances into regions of low-dose range where the dissolution rate is assumed too high.

2) Additional Parameters Describing Resist Dissolution: Investigations in X-ray lithography concerning the development process indicate that it is difficult to determine the relationship of dissolution rate and deposited dose only based on one curve chart as shown in Fig. 6. In reality, the relationship of dissolution rate and deposited dose is influenced by a large number of parameters related to chemical reaction at the liquid (GG-developer) - solid (PMMA) interface, e.g., the PMMA molecule weight, developer temperature, and the development apparatus [21]. In the case of our development process with a magnetic stirrer, the transport of material by convection supplementarily contributes to the dissolution rate [22], [23]. Development of exposed resist requires the dissolution of PMMA fragments at the interface, as well as the transport of these fragments out of the feature and away from PMMA. This means that the local development rate should then be calculated taking into account the local fragment concentration at many points along the dissolution front [7].

In X3D, the local dissolution rate at the front described by the relationship "dissolution rate as a function of dose" is a function of only one parameter. The observed results shown in Fig. 10 suggest that difference between the simulation results and measurements cannot be explained by sole integration of an error along time. The discrepancy between simulation and measurement could result from ignoring the weaker dependencies of the dissolution rate on secondary effects. Such secondary effects are diffusion and convection of resist fragments in a developer. The fact that variation of $8 \%$ in the developed depth (for the same development time) was observed on the stairs-like pattern with a number of identical experiments also supports this consideration.

Therefore, two main points are identified to get a more accurate prediction of the development profile with progressing development time. First, it is required to more accurately determine the dissolution rate, especially in the low-dose region. Second, for more sophisticated 3-D microstructures, the dissolution process in the development simulation should take into account diffusion and convection of resist fragments in the developer as described above. Improvement of the development module of X3D implementing these two points is future work.

\section{CONCLUSION}

We have developed a new simulation system covering the complete process of $\mathrm{M}^{2} \mathrm{DXL}$. From the comparison of simulation results to measurements of the stairs-like dose deposition pattern by $\mathrm{M}^{2} \mathrm{DXL}$, we confirmed the validity of our simulation tool X3D in predicting 3-D microstructures. Although there is the slight disagreement with experiments on stairs-like pattern by $\mathrm{M}^{2} \mathrm{DXL}, \mathrm{X} 3 \mathrm{D}$ reached a state where it can simulate 3-D microstructures and complement difficult experimental work on $\mathrm{M}^{2} \mathrm{DXL}$ technique. In future work, we intend to more precisely quantify dissolution rate as a function of dose in the currently inaccurate dose region. Also, we intend to improve the development module of X3D by taking into account the diffusion and convection of resist fragments in developer.

\section{ACKNOWLEDGMENT}

The authors would like to thank Y. Yamamoto of Ritsumeikan University SR Center for advice on the SR source calculation and SR center staffs for assistance in "AURORA" operation.

\section{REFERENCES}

[1] O. Tabata, K. Terasoma, N. Agawa, and K. Yamamoto, "Moving mask LIGA (M ${ }^{2}$ LIGA) process for control of side wall inclination," in Proc. 12th Int. Conf. on Micro Electro Mechanical Systems, FL, Jan. 1999, pp. 252-256. 
[2] S. Sugiyama, S. Khumpuang, and G. Kawaguchi, "Plain-pattern to cross-section transfer (PCT) technique for deep X-ray lithography and applications," J. Micromech. Microeng., vol. 14, pp. 1399-1404, 2004.

[3] D. Y. Oh, K. Gil, S. S. Chang, D. K. Jung, N. Y. Park, and S. S. Lee, "A tetrahedral three-facet micro mirror with the inclined deep X-ray process," Sens. Actuators A, Phys., vol. A 93, pp. 157-161, 2001.

[4] O. Tabata, N. Matsuzuka, T. Yamaji, H. You, J. Minakuchi, and K. Yamamoto, "Fabrication of 3-dimensional microstructures using moving mask deep X-ray lithography ( $\left.\mathrm{M}^{2} \mathrm{DXL}\right)$," in Proc.14th Int. Conf. on Micro Electro Mechanical Systems, Interlaken, Switzerland, Jan. 2001, pp. 94-97.

[5] O. Tabata, N. Matsuzuka, T. Yamaji, S. Uemura, and K. Yamamoto, “3-D fabrication by moving mask deep X-ray lithography ( $\mathrm{M}^{2} \mathrm{DXL}$ ) with multiple stages," in Proc. 15th Int. Conf. on Micro Electro Mechanical Systems, Las Vegas, NV, Jan. 2002, pp. 180-183.

[6] P. Meyer, J. Schulz, and L. Hahn, "DoseSim: Microsoft-Windows graphical user interface for using synchrotron X-ray exposure and subsequent development in the LIGA process," Rev. Sci. Instrum., vol. 74, no. 2, pp. 1113-1119, 2003

[7] S. K. Griffiths, "Fundamental limitations of LIGA X-ray lithography: Sidewall offset, slope and minimum feature size," J. Micromech. Microeng., vol. 14, pp. 999-1011, 2004.

[8] P. I. Hagouel, "Blazed diffraction gratings fabricated using X-ray lithography: Fabrication, modeling and simulation," Microelectron. Rel., vol. 43, pp. 249-258, 2003.

[9] S. B. Bollepalli, M. Khan, and F. Cerrina, "Modeling image formation in layered structures: Application to X-ray lithography," in Proc. 1st Int. Conf. on Modeling and Simulation of Microsystems Semiconductors Sensors and Actuators, Santa Clara, CA, Apr. 1998, pp. 53-58.

[10] N. Matsuzuka, Y. Hirai, S. Hafizovic, J. G. Korvink, and O. Tabata, "Effect of development process in moving mask deep X-ray lithography for 3-D microfabrication," in Proc. 5th Int. Workshop on High Aspect Ratio Micro-Structure Technology, Monterey, CA, Jun. 2003, pp. $59-60$.

[11] S. Hafizovic, Y. Hirai, O. Tabata, and J. G. Korvink, "X3-D: 3-D X-ray lithography and development simulation for MEMS," in Proc.12th Int. Conf. on Solid-State Sensors Actuators and Microsystems, Boston, MA, Jun. 2003, pp. 1570-1573.

[12] J. A. Sethian, Level Set Methods and Fast Marching Methods, 2nd ed. Cambridge, U.K.: Cambridge University Press, 1999.

[13] F. J. Pantenburg, S. Achenbach, and J. Mohr, "Influence of developer temperature and resist material on the structure quality in deep X-ray lithography," J. Vac. Sci. Technol., vol. B 16, pp. 3547-3551, 1998.

[14] M. X. Tan, M. A. Bankert, S. K. Griffiths, A. Ting, D. R. Boehme, S. Wilson, and L. M. Balser, "PMMA development studies using various synchrotron sources and exposure conditions," in Proc. SPIE Symposium on Materials and Device Characterization in Micromachining, vol. 3512, Santa Clara, CA, Sep. 1998, pp. 262-270.

[15] G. Margaritondo, Introduction to Synchrotron Radiation. New York: Oxford University Press, 1980, ch. 2.2.

[16] Z. Liu, F. Bouamrane, M. Roulliay, R. K. Kupka, A. Labeque, and S. Megterty, "Resist dissolution rate and inclined-wall structures in deep X-ray lithography," J. Micromech. Microeng., vol. 8, pp. 293-300, 1998.

[17] S. Osher and J. A. Sethian, "Fronts propagating with curvature-dependent speed: Algorithms based on Hamilton-Jacobi formulations," $J$. Comp. Phys., vol. 79, pp. 12-49, 1988.

[18] Y. Hirai, S. Tomida, K. Ikeda, M. Sasago, M. Endo, S. Hayama, and N. Nomura, "Three-dimensional resist process simulator PEACE (photo and electron beam lithography analyzing computer engineering system)," IEEE Trans. Comput.-Aided Des., vol. 10, no. 6, pp. 802-807, 1991.

[19] Y. Hirai, S. Hafizovic, J. G. Korvink, and O. Tabata, "Measurement of PMMA dissolution rate and system calibration for predictive 3-D simulation of moving mask deep X-ray lithography," in Proc. 5th Int. Workshop on High Aspect Ratio Micro-Structure Technology, Monterey, CA, Jun. 2003, pp. 37-38.

[20] - "Process simulation system for 3-D X-ray lithography and development" (in Japanese), IEEJ Trans. SM, vol. 123, no. 9, pp. 368-375, 2003

[21] P. Meyer, A. El-Kholi, and J. Schulz, "Investigations of the development rate of irradiated PMMA microstructures in deep X-ray lithography," Microelectron. Eng., vol. 63, pp. 319-328, 2002.
[22] J. Zanghellini, A. El-Kholi, and J. Mohr, "Development behavior of irradiated microstructures," Microelectronic Engineering, vol. 35, pp. 409-412, 1997.

[23] S. K. Griffiths and R. H. Nilson, "Transport limitations on development times of LIGA PMMA resists," Microsyst. Technol., vol. 8, pp. 335-342, 2002 .

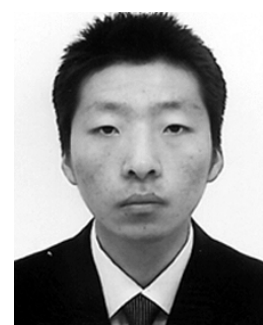

Yoshikazu Hirai was born on August 2, 1979. He received the B.S. and M.S. degrees from Ritsumeikan University, Shiga, Japan, in 2002 and 2004, respectively. He is currently working toward the Ph.D. degree in the Department of Micro Engineering at the Kyoto University, Kyoto, Japan.

Mr. Hirai is a Student Member of the Institute of Electrical Engineers of Japan and a Member of the Japan Society of Mechanical Engineers.

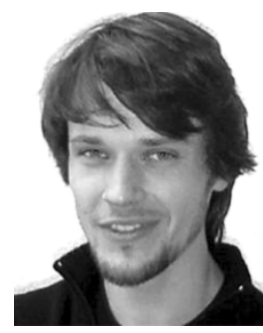

Sadik Hafizovic received the diploma degree in microsystem technology from IMTEK, University of Freiburg, Germany, in 2002. He worked on the corresponding diploma thesis in the Tabata Laboratories at the Ritsumeikan University, Japan. Since 2002, he is at the Physical Electronics Laboratory at the ETH Zurich in Switzerland, where he is currently working toward the Ph.D. degree in electrical engineering.

The focus of his research activities is on atomicforce microscopy and CMOS-based microelectrode arrays for neuron interfaces.

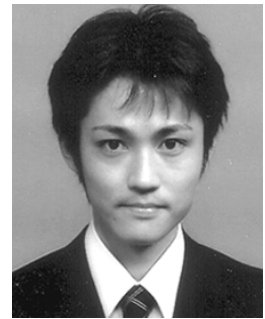

Naoki Matsuzuka was born on November 24 1977. He received the B.S. and M.S. degrees from Ritsumeikan University, Shiga, Japan, in 2000 and 2002 , respectively. He is currently working toward the $\mathrm{Ph} . \mathrm{D}$. degree at the same university.

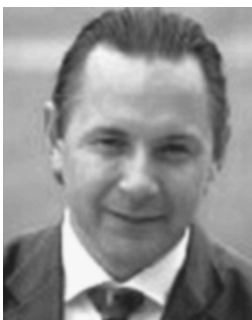

Jan G. Korvink received the M.Sc. degree in computational mechanics from the University of Cape Town, South Africa, in 1987 and the Ph.D. degree in applied computer science from the ETH Zurich, Switzerland, in 1993.

After his graduate studies, he joined the Physical Electronics Laboratory of the ETH Zurich, where he established and lead the MEMS Modeling Group. This was followed by a move in 1997 to the Alber Ludwig University in Freiburg, Germany, where he holds a Chair position in microsystem technology and runs the Laboratory for Microsystem Simulation. Currently, he is Dean of the Faculty of Applied Science. He has written more that 130 journal and conference papers in the area of microsystem technology, and co-edits the review journal Applied Micro and Nanosystems. His research interests cover the modeling and simulation and low cost fabrication of microsystems. 


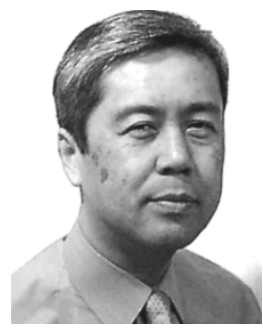

Osamu Tabata (M'89-SM'01) was born in 1956. He received the M.S. and Ph.D. degrees from Nagoya Institute of Technology, Nagoya, Japan, in 1981 and in 1993, respectively.

Since 1981, he had been with the Toyota Central Research and Development Laboratories, Inc., Aichi, Japan. In 1996, he joined the Department of Mechanical Engineering, Ritsumeikan University, Shiga, Japan. From September to December 2000, he was a guest Professor of Institute of Microsystem Technology, University of Freiburg, Germany and from January to March 2001, he was a guest Professor of ETH Zurich, Switzerland. In 2003, he joined the Department of Mechanical Engineering, Kyoto University, Kyoto, Japan. From April 2005, he is a Professor at the Department of Micro Engineering, Kyoto University, Kyoto, Japan. He is currently engaged in the research of micro/nano process, LIGA process, MEMS and micro/nano system synthetic engineering. At new position, he started the research to realize a unique and novel nanosystem by assembling the various functional components such as a microchip, a particle, a microcapsule, a cell, etc., with sizes ranging from the nanometer to micrometer scale on a few $\mathrm{mm}$ square MEMS substrate. This technology is termed SENS (synthetic engineering for nanosystems), and experimental and theoretical research on the establishment of SENS is pursued.

Dr. Tabata is an Associate Editor of Journal of MicroelectroMECHANICAL SYSTEMS and an editorial board member of Sensors and Actuators. Also, he is a Program Committee Member of many International Conferences. He was honored with the Science News Award for research in "Monolithic pressure-flow sensor" in 1987, Presentation Paper Award for research in "Anisotropic etching of silicon in TMAH solutions" in 1992, R\&D 100 Award for research in "Thin film Young's modulus measurement apparatus" in 1993 and R\&D 100 Award for research in "Thin film Tensile Tester" in 1998, Best Poster Award of 19th Sensor Symposium on Sensors, Micromachines, and Applied Systems for "Determination of Optimal Mask Movement Pattern for Moving Mask Deep X-Ray Lithography" in 2002, Best Patent Award from Ritsumeikan University for "Material Processing and its apparatus using X-ray Lithography" in 2004. He is a member of the Institute of Electrical Engineers of Japan, the Japan Society of Mechanical Engineers. 